\title{
AVALIAÇÃO DA SEGURANÇA DA PREPARAÇÃO DE MACARRÃO EM SERVIÇO DE REFEIÇÃO TRANSPORTADA DE COZINHA INDUSTRIAL DO DISTRITO FEDERAL ${ }^{1}$
}

\author{
Alessandra Sacramento dos Santos Machado \\ SENAI/DF, e-mail: alessandrassm@gmail.com \\ Andréa Clara Spoladore Damian \\ Consultora autônoma, e-mail: andreasdamian@gmail.com \\ Katherine Helena Oliveira de Matos \\ SENAI/SC, e-mail: katherine@sc.senai.br
}

\begin{abstract}
RESUMO: Foi realizado um estudo para avaliar a segurança da preparação do macarrão produzido em cozinha industrial, especializada em refeições transportadas, no atendimento aos requisitos estatutários vigentes aliados aos critérios gastronômicos necessários para a preparação. Foi avaliado o perfil de perigos da matéria-prima, as massas alimentícias, com análises microbiológicas com a pesquisa de Bacillus cereus, Coliformes a $45^{\circ} \mathrm{C} / \mathrm{g}$, Estafilococus coagulase positiva/g, Salmonella sp, bolores e leveduras e presença de matérias macroscópicas e microscópicas prejudiciais à saúde humana. $\mathrm{O}$ acompanhamento do processo de preparo do macarrão na cozinha industrial, coleta de amostras nas diversas etapas de produção e a realização de análises microbiológicas destas amostras possibilitou avaliar a segurança da preparação elaborada. Foi realizada visita ao fabricante da massa alimentícia para identificar o processo de fabricação e medidas de controle sanitário adotadas e proposto o preparo e distribuição de macarrão com molho agregado. Os resultados revelaram que as formas adotadas para o preparo e distribuição da preparação se demonstraram eficazes na manutenção da segurança dos produtos mesmo sendo considerados valores de referência abaixo dos exigidos pelas legislações vigentes.
\end{abstract}

Palavras-chave: Massa alimentícia. Refeição transportada. Segurança de alimentos.

1 Trabalho de Conclusão de Curso do MBA em Gestão para Segurança de Alimentos, SENAlsc. 


\section{INTRODUÇÃO}

A evolução do mercado de trabalho, principalmente nos grandes centros, gerada pela abertura econômica e reforçado pela inserção cada vez mais pujante das mulheres, tem levado cada vez mais o brasileiro a fazer as suas refeições fora de seus domicílios. Isto se reflete principalmente no crescimento diretamente proporcional de estabelecimentos destinados a suprir essa demanda.

No ano de 2006 o mercado de refeições coletivas, como um todo, forneceu 7,0 milhões de refeições/dia, movimentou uma cifra de 7,5 bilhões de reais, ofereceu 175 mil empregos diretos, consumiu diariamente um volume de 3,0 mil toneladas de alimentos e representou para os governos uma receita de 1 bilhão de reais entre impostos e contribuições. (ASSOCIAÇÃO BRASILEIRA DAS EMPRESAS DE REFEIÇÕES COLETIVAS, 2008)

O sistema de refeições descentralizadas ou transportadas caracteriza-se pela distância entre os locais de produção das preparações de um cardápio e de distribuição ou consumo das mesmas. Tal sistema permite o fornecimento de refeições onde não há estrutura apropriada para sua produção.

O conceito básico de cozinha industrial é o fornecimento de refeições prontas. Os serviços oferecidos dependem basicamente do mercado que se pretende atingir. Existe certa controvérsia com relação à aplicação do termo cozinha industrial. Alguns o aplicam para designar as cozinhas industriais, surgindo outras denominações, como cozinha hospitalar, para hospitais; cozinha comercial, para hotéis e restaurantes.

No Brasil, ganha campo o segmento dos semi-prontos - alimentos frescos para consumo em curto prazo, que requerem pouco tempo de preparo, criando-se então, um nicho de fornecimento de comida pronta, com empresas oferecendo itens individuais, refeições completas ou por encomenda.

Aliado a isso, empresas de todos os tamanhos tornam-se os clientes das cozinhas industriais, pois procuram, cada vez mais, oferecer refeições saudáveis para os empregados. Trata-se de um investimento na saúde da equipe, mas que também é salutar para o negócio. Afinal, um funcionário bem alimentado tem mais disposição para o trabalho e menor probabilidade de adoecer e ter que ficar afastado do serviço.

Dentre as várias opções de guarnição, o macarrão, nas mais diversas modalidades de preparo, configura um dos alimentos de maior preferência pela clientela e, por este motivo, apresenta uma das maiores incidências no cardápio em comparação às demais opções de guarnição.

O estabelecimento de critérios de tempo e temperatura nas várias etapas do processo de produção de alimentos constitui base para a segurança de alimentos, considerando a necessidade de constante aperfeiçoamento das ações de controle sanitário na área 
visando a proteção da saúde da população e a necessidade de elaboração de requisitos higiênico-sanitários gerais para serviços de alimentação face à ocorrência de doenças transmitidas por alimentos cada vez mais freqüentes.

Percebe-se que refeição transportada é problemática por fatores característicos do próprio processo. Há que se ter muito cuidado técnico no planejamento, no preparo, na montagem e no transporte dos alimentos para que se possa garantir a qualidade nutricional, sensorial, microbiológica e físico-química dos produtos. Um dos pontos críticos está na manutenção da temperatura dos alimentos durante o transporte e espera para distribuição de forma a impedir a contaminação dos mesmos devido, principalmente, à multiplicação de microorganismos que tenham resistido à cocção ou à geminação dos microorganismos esporulados.

Assim, é um desafio produzir a preparação "macarrão" em cozinhas industriais cujo serviço é descentralizado. Foram várias as ocorrências de fornecimento da preparação macarrão com temperatura inadequada ou com características sensoriais insatisfatórias. Atas de reunião entre a CPA e clientes registram um produto pegajoso, com textura demasiadamente mole e temperaturas que variam entre $36^{\circ} \mathrm{C}$ e $49^{\circ} \mathrm{C}$. Tais condições resultam em devoluções do alimento e substituições por outros produtos como farofa e batata palha.

Diante das várias barreiras operacionais e exigência por parte da clientela, a opção das empresas é a retirada da preparação do cardápio, o que contribui para a sua monotonia, e furta o comensal de um alimento importante sob o ponto de vista nutricional e cultural.

\subsection{Objetivo geral}

Avaliar o grau de segurança da preparação macarrão produzida por cozinha industrial especializada em refeição transportada.

\subsection{Objetivos específicos}

a) Avaliar processo de preparo e distribuição do macarrão;

b) Avaliar se o macarrão preparado pela empresa está em conformidade com os padrões microbiológicos preconizados pela RDC n 12/2001;

c) Desenvolver o processo de produção de macarrão com molho agregado, elaborar fluxograma de preparo e validar o fluxograma;

d) Simular a distribuição do macarrão junto aos clientes;

e) Analisar o grau de aceitação da preparação;

f) Validar o processo desenvolvido; 
g) Re-introduzir no cardápio da cozinha industrial a preparação macarrão se o produto produzido pelo método tradicional ou alternativo for aprovado nos testes microbiológicos e sensoriais.

\section{MATERIAL E MÉTODOS}

A coleta de dados foi realizada no período de Fevereiro a Julho de 2008. Amostras das massas alimentícias secas tipo espaguete e parafuso, com ovos, foram enviadas a laboratórios especializados. As massas avaliadas são as mesmas adquiridas e utilizadas pelo serviço de alimentação, de marca única ao longo dos últimos 3 anos.

As massas parafuso e espaguete foram submetidas às análises: contagem de Bacillus cereus, Coliformes a $45^{\circ} \mathrm{C} / \mathrm{g}$, Salmonella spp / 25 g, Staphylococcus coag. positiva/g, bolores e leveduras e sujidades (exames macroscópico e microscópico).

Para a pesquisa de Bacillus cereus, Coliformes a $45^{\circ} \mathrm{C} / \mathrm{g}$, Staphylococcus coag. Positiva/g e bolores e leveduras a metodologia utilizada foi a Instrução Normativa SDA no.62, MAPA/2003 - Métodos Analíticos Oficiais para Análises Microbiológicas para Controle de Produtos de Origem Animal e Água. A pesquisa de Salmonella spp / $25 \mathrm{~g}$ foi realizada pelo método 996.08 - Isolamento e Identificação de Salmonella spp da Association of Official Analytical Chemists (AOAC).

Para a análise de sujidades e exame macroscópico foi utilizado o Approved Methods of the American Association of Cereal Chemists (AACC, 2000). No exame microscópico foi utilizado o Official Methods of Analysis of AOAC International.

As características tecnológicas das massas alimentícias foram avaliadas através das determinações de cor, umidade, teste de cozimento (incluindo os parâmetros tempo de cozimento, perda de sólidos, aumento de volume e aumento de peso) e textura (pegajosidade e firmeza). Os testes de umidade e cozimento foram repetidos 3 (três) vezes, pegajosidade e firmeza tiveram 15 (quinze) repetições e o teste de cor contou com 8 (oito) repetições.

A umidade foi realizada pelo método 44-15 A; o teste de cozimento pelo método 66-50; a textura e parâmetro firmeza pelo método 116-50. Estas metodologias são do American Association Of Cereal Chemists (AACC, 2000). Os testes de pegajosidade e colorimetria foram realizados pelos métodos MA-CH.019 e MA-CH.013, respectivamente.

A determinação do teor de umidade em massa alimentícia é feita visando controlar a eficiência do processo de secagem e verificar se o produto obtido apresenta boas condições de armazenamento.

O teste de cozimento fornece informações de como o produto se comporta durante a cocção, considerando a perda de nutrientes na água. Quanto ao tempo de cozimento, este varia em função do formato e tamanho das massas. 
A textura do produto cozido, no quesito firmeza, diz respeito à força máxima necessária para cortar o macarrão. Para a realização desta avaliação foi utilizado o equipamento texturômetro modelo TA-XT2i da marca Stable Micro Systems. A pegajosidade da massa é definida como a força máxima necessária para separar o equipamento da superfície da amostra após um tempo de contato. Quanto maior essa força, maior a pegajosidade da amostra.

\section{RESULTADOS E DISCUSSÃO}

\subsection{IDENTIFICAÇÃO E CARACTERIZAÇÃO DOS PERIGOS DA MATÉRIA-PRIMA CHAVE}

A tabela 1 apresenta os resultados da avaliação microbiológica das massas alimentícias:

Tabela 1 - resultados da avaliação microbiológica das massas alimentícias:

\begin{tabular}{|c|c|c|c|}
\hline & & rrão Espaguete & Macarrão \\
\hline & Padrão ${ }^{1}$ & Resultado ${ }^{2}$ & Resultado ${ }^{2}$ \\
\hline Bacillus cereus & $5 \times 10^{3}$ & $<10$ & $<10$ \\
\hline Coliformes a $45^{\circ} \mathrm{C} / \mathrm{g}$ & $10^{2}$ & $<10$ & $<10$ \\
\hline Coliformes totais & - & $<10$ & $<10$ \\
\hline Salmonela spp / $25 \mathrm{~g}$ & Ausência & Ausência & Ausência \\
\hline Staphylococcus coag. positiva/g & $5 \times 10^{3}$ & $<10$ & $<10$ \\
\hline Bolores e leveduras & - & $<10$ & $<10$ \\
\hline Exame macroscópico & Ausência & Ausência & Ausência \\
\hline Exame microscópico & Ausência & Ausência & Ausência \\
\hline${ }^{1}$ Conforme RDC 275/02 - Anvisa (BRASI & 2001). & & \\
\hline${ }^{2}$ Valores expressos em UFC/g & & & \\
\hline
\end{tabular}

Os resultados obtidos e demonstrados acima sugerem que as matérias-primas não apresentam perigos significativos uma vez que os valores encontrados dos microrganismos pesquisados são menores quando comparados aos estabelecidos pela legislação aplicável. Este resultado, aliado à aplicação de ações que evitem a ocorrência de contaminações cruzadas no decorrer das demais etapas de preparo do macarrão, pode contribuir para a produção de um produto final que esteja em conformidade com os parâmetros de seguranças. 


\subsection{CARACTERIZAÇÃO SENSORIAL DA MATÉRIA-PRIMA}

A tabela 2 apresenta os resultados da avaliação sensorial das matérias-primas:

Tabela 2 - Resultados da avaliação sensorial das matérias-primas

\begin{tabular}{l|c|c|} 
& Macarrão Espaguete & Macarrão \\
& & Parafuso \\
\hline & Resultado & Resultado \\
\hline Umidade (\%) & 11,54 & 11,62 \\
\hline Cor & 92,26 & 86,26 \\
\hline Textura: & & \\
\hline Firmeza (gf) & 276,7 & 466,0 \\
\hline Pegajosidade (gf) & 192,7 & - \\
\hline Teste de cozimento: & & 6 \\
\hline Tempo de cozimento (minutos) & 8 & 4,4 \\
\hline Perda de sólidos (\%) & 4,97 & 133,3 \\
\hline Aumento de volume (\%) & 244,4 & 132,0 \\
\hline Aumento de peso (\%)
\end{tabular}

Fonte: Instituto de Tecnologia de Alimentos (2008).

No quesito umidade, espera-se que valores estejam sempre abaixo de $13 \%$. Nesta faixa o produto teoricamente está isento de problemas microbiológicos. Os produtos analisados apresentaram umidade menor que $12 \%$.

No teste de cozimento o tempo de cozimento resultante da análise é bastante próximo do tempo praticado durante o tratamento térmico na CPA que é de 5 a 6 minutos em média.

Segundo os critérios de Hummel (1966), perdas de sólidos de até 6\% são características de massas de trigo de qualidade muito boa, até $8 \%$ de massas de média qualidade e valores iguais ou superiores a $10 \%$ são característicos de massas de qualidade ruim. Donnely (1979) também considera $8 \%$ como o valor máximo aceitável para a perda de sólidos na água de cozimento. A perda nas massas tipo espaguete e parafuso se mantiveram abaixo de $5 \%$, evidenciando a qualidade da matéria-prima empregada.

O fato de a massa tipo espaguete ter apresentado resultados melhores para os aumentos de peso e volume pode ser justificado por sua maior superfície de contato possibilitando uma maior absorção de água. 


\subsection{ANÁLISE DO PROCESSO DE PRODUÇÃO TRADICIONAL DA PREPARAÇÃO MACARRÃO NA CPA}

Os gráficos abaixo apresentam o perfil microbiológico da preparação macarrão em suas diversas etapas do preparo considerando os diferentes dias de cardápio e com diferenciados molhos.

Os valores dos eixos Y estão expressos em UFC/g.

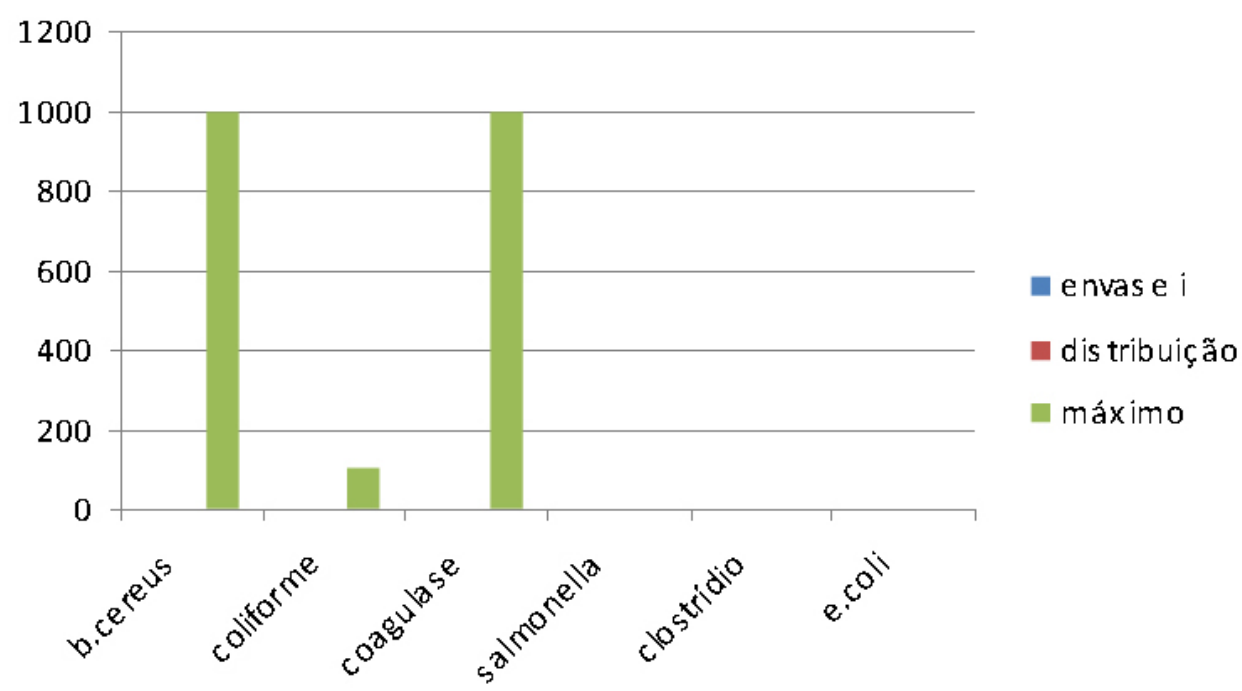

Figura 1 - Espaguete à chinesa* em 12/02/2008.

Fonte: Fonte:Lab. Hig. Alimentos - UnB

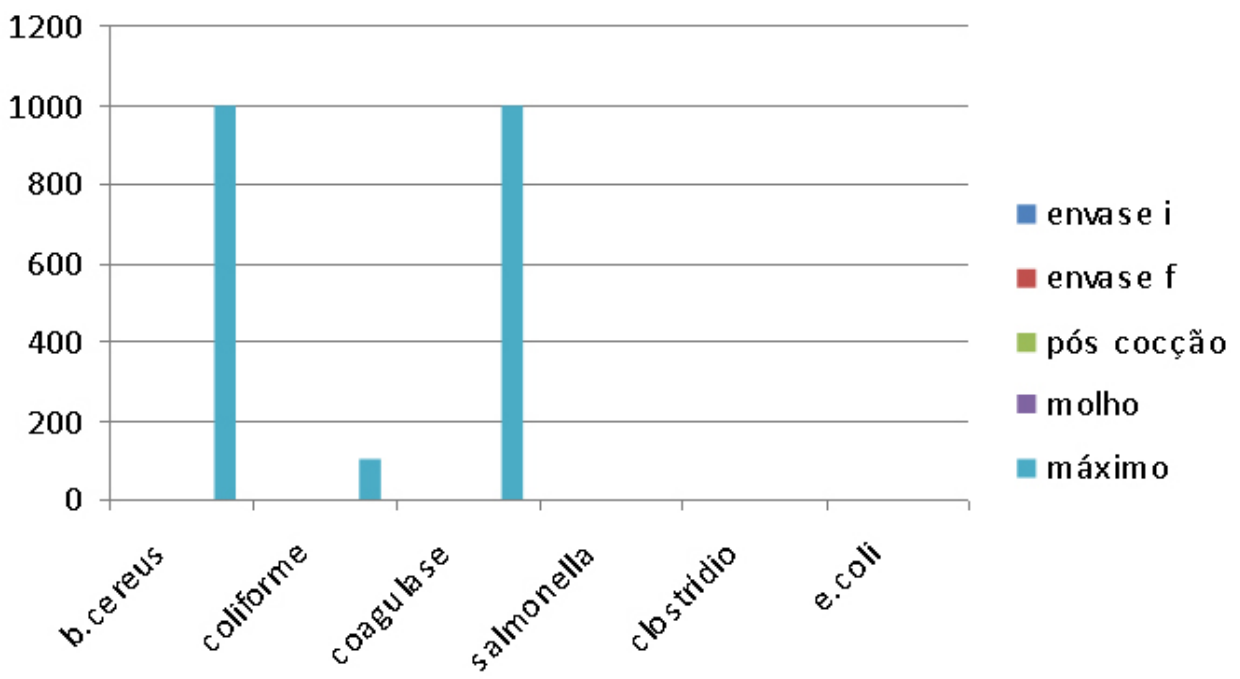

* Espaguete à chinesa: molho shoyo e broto de bambu

Figura 2 - Espaguete à chinesa em 01/04/2008.

Fonte: Lab. Hig. Alimentos - Unb 
O resultado das 192 análises microbiológicas do processo tradicional de preparo do macarrão evidencia a segurança das preparações nas diferentes etapas do processo e mesmo com a temperatura no momento do envase (entre $44^{\circ} \mathrm{C}$ e $53^{\circ} \mathrm{C}$ ) e distribuição (entre $42,5^{\circ} \mathrm{C}$ e $52^{\circ} \mathrm{C}$ ), abaixo da ideal, em um intervalo médio de 4 horas após o envase.

\subsection{ANÁLISE DO DESENVOLVIMENTO DO PROCESSO ALTERNATIVO DE PREPARO DO MACARRÃO}

As tabelas 3 e 4 demonstram os resultados das simulações de preparo e distribuição de macarrão com molho agregado.

Tabela 3 - Simulação com Macarrão Espaguete

\begin{tabular}{l|c|c|c|}
\hline Parâmetros & Simulação 1 & Simulação 2 & Simulação 3 \\
\hline Apresentação & Massa grudada & Massa grudada & Massa solta \\
\hline Textura da massa & Muito cozida & Mole & Mole \\
\hline Absorção de molho & Normal & Normal & Normal \\
\hline Temperatura & $49^{\circ} \mathrm{C}$ & $47^{\circ} \mathrm{C}$ & $54^{\circ} \mathrm{C}$
\end{tabular}

Simulação 1: Cocção por 6 minutos, resfriamento em água fria corrente

2: Cocção por 5 minutos, resfriamento em água fria corrente

3: Cocção por 4 minutos, sem resfriamento e com enxágüem em água quente corrente

Tabela 4 - Simulação com Macarrão Parafuso

\begin{tabular}{l|c|c|c|}
\hline Parâmetros & Simulação 1 & Simulação 2 & Simulação 3 \\
\hline Apresentação & Massa grudada & Massa grudada & Massa grudada \\
\hline Textura da massa & Muito cozida & Mole & Mole \\
\hline Absorção de molho & Normal & Normal & Normal \\
\hline Temperatura & $45^{\circ} \mathrm{C}$ & $48^{\circ} \mathrm{C}$ & $55^{\circ} \mathrm{C}$ \\
\hline
\end{tabular}

Simulação 1: Coç̧ão por 6 minutos, resfriamento em água fria corrente

2: Cocção por 5 minutos, resfriamento em água fria corrente

3: Cocção por 4 minutos, sem resfriamento e com enxágüem em água quente corrente 
Os resultados das simulações de preparo e distribuição de macarrão espaguete e parafuso com molho agregado, tabelas 1 e 2 , indicaram que ainda que o molho agregado esteja em temperatura demasiadamente quente não é suficiente para que a preparação atinja a temperatura ideal com resfriamento em água fria.

A cocção por 6 e 5 minutos em ambas as massas contribuiu para um produto final de apresentação ruim e textura variando de muito cozida a mole. Embora o resfriamento tenha sido em água fria, a adição de molho quente e o acondicionamento em caixa isotérmica por 4 horas possibilita criar condições similares ao cozimento.

O tratamento térmico por 4 minutos, sem resfriamento e enxágüe em água quente corrente objetivando manter a cadeia térmica foi benéfica apenas para o macarrão tipo espaguete com o resultado de massa solta. Ainda, houve um aumento importante da temperatura, da ordem de $7^{\circ} \mathrm{C}$ para o espaguete e o parafuso se comparados à simulação 2 , mas distante da temperatura alvo de $60^{\circ} \mathrm{C}$.

O não atendimento ao requisito da temperatura ideal aliado ao produto com características organolépticas insatisfatórias foi determinante para interromper os testes.

\section{CONCLUSÃO}

O processo de preparo do macarrão na CPA é realizado em consonância com os requisitos previstos nas legislações sanitárias e sistemas de qualidade e segurança de alimentos vigentes.

As análises microbiológicas evidenciam que a preparação do macarrão no modelo tradicional, nos diversos momentos e sob diferentes formulações de cardápio, está em conformidade com os padrões microbiológicos preconizados pela RDC $\mathrm{n} \cong$. . 12/2001.

Os microrganismos pesquisados apresentam valores significativamente menores quando comparados com os valores máximos permitidos, mesmo com as temperaturas de envase e distribuição estarem em sua maioria abaixo de $60^{\circ} \mathrm{C}$.

O processo alternativo de preparo e distribuição do macarrão com molho agregado não obteve êxito. A falta de alcance da temperatura desejada e dos padrões gastronômicos mínimos necessários nas preparações elaboradas, impossibilitou a execução das atividades seguintes de elaboração e validação de fluxograma, simulação da distribuição aos clientes, análise do grau de aceitação e validação deste processo.

O processo alternativo teria melhor resultado se a massa alimentícia à base de ovos fosse substituída pela massa do tipo grano duro, reconhecidamente mais resistente na cocção e demais etapas consecutivas. Entretanto, esta massa apresenta custo muito elevado para o padrão das refeições fornecidas pela cozinha industrial e, portanto, torna-se financeiramente inviável a sua aquisição e uso. 
Conclui-se que há subsídios suficientes que permitem a re-inserção no cardápio desta cozinha industrial a preparação macarrão elaborados a partir do método tradicional uma vez que esta foi aprovada nos testes microbiológicos e sensoriais.

Os estudos realizados permitem a prática de produção e distribuição da preparação macarrão na modalidade refeição transportada em temperatura abaixo de $60^{\circ} \mathrm{C}$ na cozinha industrial estudada e sob os critérios descritos. Este trabalho não teve a pretensão de propor mudança na legislação nacional, mas sim fomentar os procedimentos de um estabelecimento de alimentação em particular.

É importante ressaltar que esta análise foi realizada apenas com a marca de macarrão atualmente utilizada pela cozinha industrial. Em caso de mudança de matéria-prima ou de procedimentos, é de suma importância que os estudos sejam replicados e a implementação de procedimento de exigência de fornecimento de laudo laboratorial da matéria-prima junto ao fornecedor nos processos de aquisição do produto constitui o primeiro passo. 


\title{
SAFETY EVALUATION OF THE PASTA IN SPECIALIZED INDUSTRIAL FOOD SERVICE IN TRANSPORTED MEALS IN DISTRITO FEDERAL
}

\begin{abstract}
A study has been conducted for the validation of safety production of pasta in specialized industrial kitchen in transported meals according to the demands of the statutory requirements in force, allied to the necessary gastronomic criteria for the preparation of the pasta. The risk rate of raw-material has been validated with microbiologic analysis with the search for Bacillus cereus, Coliforms at $45^{\circ} \mathrm{C} / \mathrm{g}$, Estafilococus coagulase positiva/g, Salmonella sp, mold and yeast and the presence of macroscopic and microscopic material harmful to the health of humans. The monitoring of the process of preparation of the pasta at the production center, the collection of samples at different stages of production, and the microbiologic analysis of these samples made it possible to validate the safety of the product made at the industrial kitchen. A visit to the dough manufacturer was made to identify the manufacturing process as well as sanitary control measures and the method for preparation and distribution of the pasta with sauce was proposed. The results revealed that the measures adopted for preparation and distribution of the food were effective in the maintenance of the safety of the products even when the reference figures demanded were below the ones required by the legislation in force.
\end{abstract}

Key-words: Pasta. Transported meal. Food safety.

\section{REFERÊNCIAS}

ASSOCIAÇÃO BRASILEIRA DAS EMPRESAS DE REFEIÇÕES COLETIVAS. História e mercado. Apresenta informações sobre o segmento de refeições coletivas. Disponível em: http://www.aberc.com.br. Acesso em 30 jul.2008.

BRASIL. ANVISA - AGÊNCIA NACIONAL DA VIGILÂNCIA SANITÁRIA. Resolução RDC no 12, de 02 de janeiro de 2001. Aprova o Regulamento Técnico sobre padrões microbiológicos para alimentos. Diário Oficial da União, Poder Executivo, de 10 de janeiro de 2001.

HUMMEL, C. Macaroni products. London: Food Trade Press, 1966. 287p. 


\section{SOBRE O(S) AUTOR(ES)}

\begin{tabular}{|l} 
Nutricionista graduada pela Universidade de Brasília (UnB) e Especialista em \\
Gestão para a Segurança de Alimentos (SENAI SC) e Nutrição Clínica (Faculdade \\
São Camilo, Rio de Janeiro). Graduada em Formação Pedagógica para \\
Formadores da Educação Profissional pela Faculdade do Sul de Santa Catarina, \\
Unisul. \\
É consultora e multiplicadora do Programa Alimentos Seguros (PAS) - Setor \\
Mesa. \\
Atuou como Nutricionista do SESI/DF na Central de Produção de Alimentos \\
Albano Franco, instrutora e consultora do SEBRAE/DF e SENAC/DF para \\
implantação de Sistemas de Gestão para a Segurança de Alimentos. Trabalhou \\
na implementação de Sistemas de Gestão para a Segurança de Alimentos em \\
diversas empresas do segmento. \\
Sacramentro dos \\
Stualmente é Supervisora Técnica das áreas Alimentos, Gestão, Design e e \\
Vestuário nas atividades voltadas para a Educação Profissional e Serviços \\
Técnicos e Tecnológicos no SENAI/DF e Especialista da Secretaria de Saúde do \\
Distrito Federal (SES/DF).
\end{tabular}

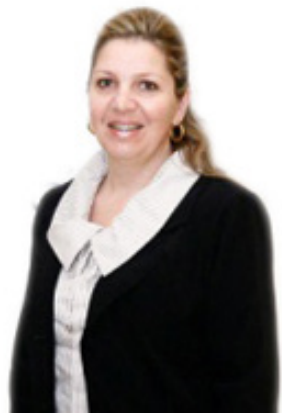

Andréa Clara Spoladore Damian
Formação: Mestrado em Ciência dos Alimentos.

Universidade Federal de Santa Catarina, UFSC, Florianópolis, Brasil

Título: Estudo comparativo das alterações de processamento no brócoli (Brassica oleracea L. var. Itálica) submetido a diferentes processos de congelamento e períodos de estocagem.

Graduação em Farmácia Bioquímica de Alimentos.

Universidade Federal de Santa Catarina, UFSC, Florianópolis, Brasil

Graduação em Pedagogia com ênfase em ensino à distância

Universidade do Sul de Santa Catarina, UNISUL, Florianópolis, Brasil

Atuação em consultoria na área de segurança de alimentos, dando enfoque a implantação de sistema de segurança de alimentos tais como Boas Práticas de Fabricação e Análise de Perigos e Pontos Críticos de Controle.

Implantação das Boas Práticas de Fabricação seguindo a metodologia do Programa Alimentos Seguros em 123 empresas do estado de Santa Catarina, implantação do sistema de segurança de alimentos APPCC (Análise de Perigos e Pontos Críticos de Controle) em 25 empresas, supervisão dos trabalhos de implantação em 750 empresas de Santa Catarina como consultora técnica estadual, através de auditoria 
Atuação em treinamentos na área e alimentos desde 2002.

Atuação em docência de graduação para os cursos de Medicina, Biologia, Nutrição, Enfermagem e Odontologia da Universidade Federal de Santa Catarina.

Professora Tutora dos cursos de MBA Gestão para a Segurança de Alimentos e Gestão para Excelência no SENAI-Florianópolis de 2007 a 2011.

Professora orientadora dos cursos de MBA Gestão para a Segurança de Alimentos e Gestão para Excelência no SENAI-Florianópolis de 2007 a 2011, atuando em mais de 24 orientações diretas e 43 bancas.

Auditora Líder da ISO 22000:2006.

Implantação de APPCC em indústria de cosmético.

Implantação de APPCC em indústria de extração de Mel.

Atuou como Coordenadora de pós graduação MBA em Gestão para Excelência e Gestão para Segurança de alimentos no SENAI-Florianópolis de 2007 a 2011.

Auditora internacional em FSSC 22000 e PAS220.

Inglês fluente.

andreasdamian@gmail.com

\begin{tabular}{|l|l|} 
& $\begin{array}{l}\text { Engenheira de Alimentos graduada pela Universidade Federal de Santa Catarina } \\
\text { (UFSC) e mestra em Engenharia de Alimentos pela Universidade Federal de } \\
\text { Santa Catarina (UFSC). Doutoranda em Engenharia de Alimentos pela mesma } \\
\text { universidade. } \\
\text { É Lead Assessor na ISO 22000:2005, consultora, auditora e multiplicadora do } \\
\text { Programa Alimentos Seguros (PAS) - Setor Indústria. } \\
\text { Atuou como instrutora e consultora do SENAI SC em Florianópolis para } \\
\text { implantação de Sistemas de Gestão para a Segurança de Alimentos. Foi docente } \\
\text { do curso de Pós-Graduação MBA em Gestão para Segurança de Alimentos a } \\
\text { distância e trabalhou na implementação e em auditorias internas de Sistemas } \\
\text { de Gestão de Qualidade e de Segurança de Alimentos em diversas indústrias } \\
\text { da área. Autora do livro Sistemas de Gestão de Segurança de Alimentos, em } \\
\text { 2008, publicado pelo SENAI e do livro Sistema de Qualidade em Segurança de } \\
\text { Alimentos: ABNT NBR ISO 22000:2006, Eurepgap e BRC, publicado em 2007, } \\
\text { pelo SENAI. } \\
\text { Atualmente é Coordenadora de Consultoria em Gestão do SENAI SC. }\end{array}$ \\
\hline
\end{tabular}

